REVIEW

\title{
Management of hyperthyroidism during pregnancy and lactation
}

\author{
Fereidoun Azizi and Atieh Amouzegar \\ Research Institute for Endocrine Sciences, Endocrine Research Center, Shahid Beheshti University (MC), PO Box 19395-4763, \\ Tehran 198517413, Islamic Republic of Iran
}

(Correspondence should be addressed to F Azizi; Email: azizi@endocrine.ac.ir)

\begin{abstract}
Introduction: Poorly treated or untreated maternal overt hyperthyroidism may affect pregnancy outcome. Fetal and neonatal hypo- or hyper-thyroidism and neonatal central hypothyroidism may complicate health issues during intrauterine and neonatal periods.

Aim: To review articles related to appropriate management of hyperthyroidism during pregnancy and lactation.

Methods: A literature review was performed using MEDLINE with the terms 'hyperthyroidism and pregnancy', 'antithyroid drugs and pregnancy', 'radioiodine and pregnancy', 'hyperthyroidism and lactation', and 'antithyroid drugs and lactation', both separately and in conjunction with the terms 'fetus' and 'maternal.' Results: Antithyroid drugs are the main therapy for maternal hyperthyroidism. Both methimazole (MMI) and propylthiouracil (PTU) may be used during pregnancy; however, PTU is preferred in the first trimester and should be replaced by MMI after this trimester. Choanal and esophageal atresia of fetus in MMI-treated and maternal hepatotoxicity in PTU-treated pregnancies are of utmost concern. Maintaining free thyroxine concentration in the upper one-third of each trimester-specific reference interval denotes success of therapy. MMI is the mainstay of the treatment of post partum hyperthyroidism, in particular during lactation. Conclusion: Management of hyperthyroidism during pregnancy and lactation requires special considerations and should be carefully implemented to avoid any adverse effects on the mother, fetus, and neonate.
\end{abstract}

European Journal of Endocrinology 164 871-876

\section{Introduction}

Hyperthyroidism during pregnancy is uncommon and has been reported as occurring in $0.05-3.0 \%$ of pregnancies $(1,2)$. The clinical diagnosis of hyperthyroidism may be difficult in pregnant women, as symptoms and signs of nervousness, sweating, and dyspnea; tachycardia and cardiac systolic murmur are seen in most normal pregnancies. More specific findings such as weight loss, goiter, and ophthalmopathy may suggest Graves' hyperthyroidism (3, 4). In addition, the occurrence of transient hyperthyroidism of hyperemesis gravidarum may complicate the diagnosis $(5,6)$. The diagnosis of hyperthyroidism should always be confirmed by the measurement of circulating free thyroxine $\left(\mathrm{FT}_{4}\right)$ and TSH. Serum $\mathrm{T}_{4}$ concentration (both total and free) varies during normal pregnancy and normal range values of total and $\mathrm{FT}_{4}$ and free tri-iodothyronine $\left(\mathrm{T}_{3}\right)$ as well as TSH concentrations must be developed for each specific trimester of pregnancy $(4,7)$.

Overt hyperthyroidism has well-documented adverse impacts on pregnancy outcomes $(8-10)$. Therefore, despite its rarity, recognition and proper management of hyperthyroidism during pregnancy is of utmost importance. Medical therapy is preferred by most authorities, as radioiodine is contra-indicated and thyroidectomy requires pre-treatment with antithyroid drugs and may be complicated by surgical adverse effects. However, there is no consensus regarding the best method of therapy for hyperthyroidism during pregnancy. The objective of this study is to review specific considerations in the management of hyperthyroidism during pregnancy and the post partum period.

\section{Methods}

\section{Data source}

The terms 'hyperthyroidism and pregnancy', 'antithyroid drug and pregnancy', 'radioiodine and pregnancy', 'hyperthyroidism and lactation', 'antithyroid drug and lactation', both separately and in conjunction with the terms 'fetus' and 'maternal' were used to search MEDLINE for articles published between 1980 and June 2010. All abstracts were reviewed; studies 
published in English were included if appropriately designed. The articles of abstracts meeting criteria were then reviewed to identify details of materials related to pregnancy complicated by hyperthyroidism, breastfeeding, and antithyroid drugs. The strategy used to search for articles was developed with the assistance of a research librarian at the Research Institute for Endocrine Sciences of Shahid Beheshti University of Medical Sciences.

\section{Study selection}

The following criteria were considered essential for an article to qualify for inclusion in this review:

i) Proper study design of survey, case-control and cohort studies, and clinical trials.

ii) Review articles by prominent scholars if the authors had at least four articles of their own in the list of references of review papers.

Reviewers were not blinded to the study authors' names. This resulted from the fact that we wanted to include all pertinent studies, and it was an exposure to the articles and the study authors. A deliberate strategy to limit bias was, therefore, developed. All articles were initially potential candidates for inclusion; an article was excluded if it lacked appropriate study design.

\section{Results}

\section{What are the changes in thyroid function during normal pregnancy?}

As pregnancy is a high estrogenic state, serum thyroxin binding globulin (TBG) increases and results in high serum total $\mathrm{T}_{4}$ and $\mathrm{T}_{3}$ concentrations. During the first trimester, the serum hCG level rises continuously and peaks near the end of the first trimester. The stimulating effect of hCG on the TSH receptor causes a fall in serum TSH levels in the first trimester of pregnancy $(11,12)$. Being aware of the changes regarding serum $\mathrm{FT}_{4}$, TSH and hCG levels throughout pregnancy would be helpful for interpretation of thyroid function test in hyperthyroid mothers (Fig. 1) (11).

\section{Why is the fetus of hyperthyroid mother at risk?}

TSH receptor stimulating autoantibodies are the culprits of pathogenesis of hyperthyroidism in fetus (13). The likelihood of developing fetal hyperthyroidism requiring treatment is related to the level of maternal stimulating TRAb levels, medical treatment of maternal disease. A study showed that the transplacental passage of stimulating maternal TRAb caused hyperthyroidism or Graves' disease in $1-5 \%$ of neonates who were
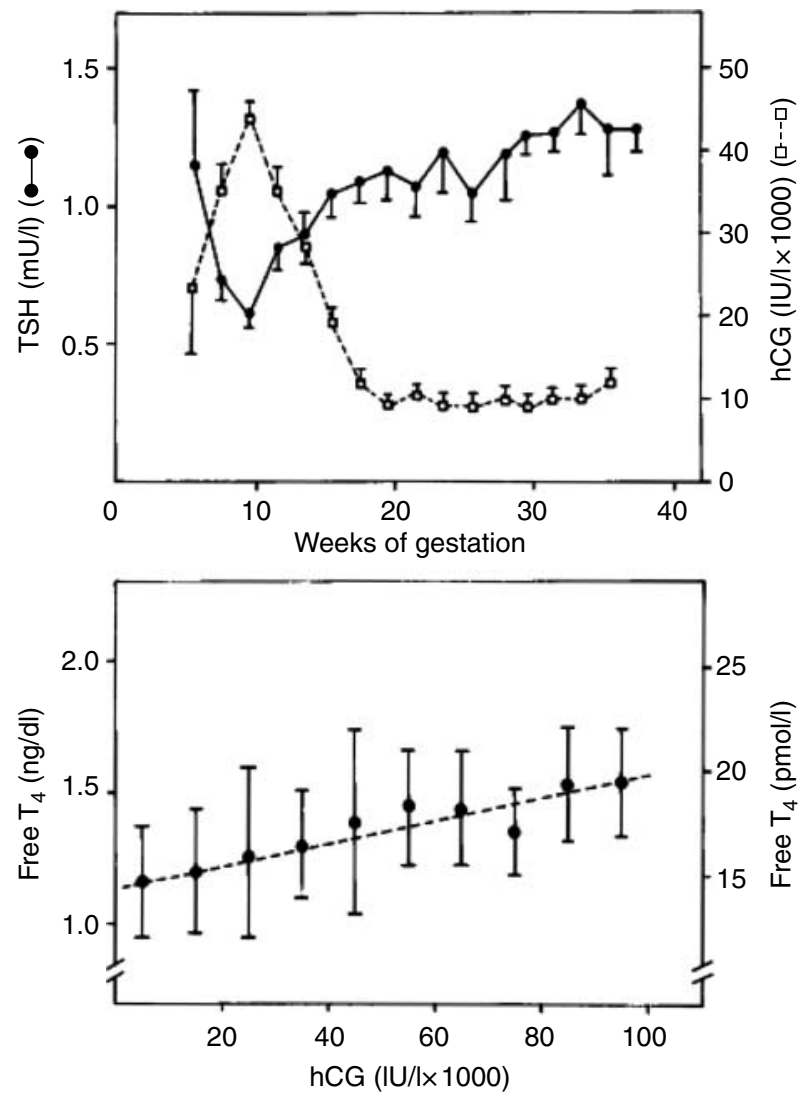

Figure 1 Alteration in serum concentration of hCG, TSH, and $\mathrm{FT}_{4}$ during pregnancy. Reproduced with permission from Glinoer $D$. The regulation of thyroid function in pregnancy: pathways of endocrine adaptation from physiology to pathology. Endocrine Reviews 1997 18 404-433. Copyright 1997, The Endocrine Society.

born to mothers with Graves' disease (14). The incidence was low because of the balance of stimulatory and inhibitory antibodies and thionamide treatment of the mothers (13). It passes the placenta and stimulates fetal thyroid and may cause fetal hyperthyroidism that manifests with fetal tachycardia (heart rate $>160$ beats/min), goiter, prematurity, heart failure, and hydrops (1-3). It occurs in 5\% of neonates of mothers with Graves' disease.

\section{What are the consequences of untreated hyperthyroidism during pregnancy?}

Low birth weight (odds ratio $(\mathrm{OR})=9.2$, 95\% confidence interval (CI) 5.5-16), prematurity $(\mathrm{OR}=16.5$, 95\% CI 2.1-130), eclampsia (OR $=4.7$, 95\% CI $1.1-$ 19.7), and risk of miscarriage are more common in untreated hyperthyroid mother than in those who are euthyroid $(9,14,15)$. The frequency of small-forgestational age infants may increase in those who remain hyperthyroid (26.7 vs $7.7 \%$ ) compared with those who were euthyroid throughout pregnancy (16). Whether the untreated Graves' disease is associated 
with congenital anomaly is a matter of debate. Some studies reported more birth defects in hyperthyroid in comparison to euthyroid women $(16,17)$.

\section{What is the best choice of treatment in pregnant patients with hyperthyroidism?}

Antithyroid drugs are the treatment of choice for hyperthyroidism during pregnancy (18). They inhibit thyroid hormone synthesis by reducing iodine organification and coupling of MIT and DIT. Methimazole (MMI), propylthiouracil (PTU), and carbimazole have been used for the treatment of hyperthyroidism during pregnancy. The pharmacokinetics of MMI is not altered in pregnancy; it has been reported that serum PTU concentrations may be lower in the third than in the first and second trimesters of gestation (19). Use of PTU should be restricted to first trimester of pregnancy, after which change to MMI is recommended (20).

Although adrenergic $\beta$-blocking agents may be used for the management of hyper-metabolic symptoms, their use should be limited to a few weeks because of possible intrauterine growth retardation (21) and, if used in late pregnancy, they may be associated with transient neonatal hypoglycemia, apnea, and bradycardia.

\section{What is the amount of placenta passage of MMI and PTU?}

All antithyroid drugs cross the placenta and may potentially affect fetal thyroid function (22).

Although PTU is more extensively bound to serum albumin than MMI and hypothetically less of it might be transferred through placenta than MMI, it has been shown that placental passage of PTU and MMI is similar. A study showed that transfer rates across the placenta were independent of the perfusate protein concentration, and this might be due to highly efficient placental extraction of the unbound drug (23). Cord PTU levels were higher than maternal concentrations in hyperthyroid pregnant patients treated with PTU until term (24). In addition, there were no differences in thyroid hormone and TSH concentrations in cord blood at birth between the MMI- and the PTU-treated newborn (25).

\section{What are the side effects and complications of MMI and/or PTU in mothers and which one should be used in maternal hyperthyroidism?}

The side effects of these drugs occur in a small number of patients taking thionamide drugs. Mostly, minor complications such as skin reactions, arthralgias, and gastrointestinal discomfort occur; however, major and sometimes life-threatening or even lethal side effects, including agranulocytosis, polyarthritis, vasculitis, and immunoallergic hepatitis, may be seen (26).
Agranulocytosis was seen in $0.35-0.4 \%$ of patients using both antithyroid drugs. Vasculitis was seen more commonly with PTU and antineutrophil cytoplasmic antibody positivity was 40 times more frequent with PTU than with MMI (27). Immunoallergic hepatitis occurs only with PTU, its frequency ranging between 0.1 and $0.2 \%$ (28). PTU-related liver failure is seen in one in every 10000 adults and one in 2000 children and on average; it occurs 3 months after initiation of PTU therapy (29), although this complication may occur at any time during PTU treatment. In severe cases, up to $25-50 \%$ fatality has been reported and liver transplantation may be required (30). Therefore, it has been advised that PTU should not be prescribed as the first-line agent in children or adults. However, due to the probability of association of fetal teratogenicity with MMI, PTU is still recommended as the drug of choice during the first trimester of pregnancy (31). Only two cases of liver failure have so far been reported with PTU in pregnancy $(32,33)$.

\section{What are the associated complications with antithyroid drugs in the fetus?}

Three types of side effects of antithyroid drugs should be considered.

Teratogenicity There are two distinct teratogenicity patterns, aplasia cutis and choanal/esophageal atresia, reported with MMI use during pregnancy, but the data are controversial. Although multiple case reports of animal studies have been published associating aplasia cutis with MMI therapy in pregnant mothers (34), no case of aplasia cutis was seen in a series of 243 pregnant women treated with MMI (35), and the occurrence of aplasia cutis with MMI did not exceed baseline rate of one in 30000 births in normal pregnancies (36). Choanal and esophageal atresia may have a higher incidence than that expected in fetuses exposed to MMI during the first trimester of gestation, OR may be as high as 18 (37-39). However, the mother's disease might be the causal factor rather than MMI treatment (40). A prospective cohort study did not show any significant difference in incidence of major anomalies or spontaneous abortions between MMI treatment and controls during pregnancy (37).

Effects on the fetal thyroid There is a lack of correlation between fetal thyroid function and maternal dosage of antithyroid drugs $(23,41,42)$. Decreased serum- $\mathrm{FT}_{4}$ in $36 \%$ of neonates is seen when the maternal serum- $\mathrm{FT}_{4}$ is in the lower two-thirds of the normal non-pregnant reference range. Maternal thyroid status is the most reliable marker, and in pregnant mothers with serum- $\mathrm{FT}_{4}$ levels in upper third of the normal range, serum- $\mathrm{FT}_{4}$ concentrations of over $90 \%$ of their neonates are within normal range $(17,23,42)$. 
Overtreatment of pregnant ladies with antithyroid drugs resulting in decreasing maternal serum- $\mathrm{FT}_{4}$ is usually accompanied by fetal hypothyroidism.

Effect on pediatric physical and mental growth No differences in thyroid function or physical and psychomotor development has been found between children born to MMI- or PTU-treated hyperthyroid mothers during pregnancy and those born to euthyroid mothers $(43,44)$.

\section{Which tests should be performed during treatment with ATD and what levels should be achieved?}

Methimazole in doses of $10-20 \mathrm{mg}$ or PTU 100-200 mg daily should be started, and after 1 month, it is desirable to adjust the doses in order to maintain maternal $\mathrm{FT}_{4} \mathrm{I}$ in the upper one-third of each trimester-specific reference interval (41) (Fig. 2). $\mathrm{FT}_{4} \mathrm{I}$ and TSH should be monitored at monthly intervals during pregnancy. Serum TSH levels of $0.1-2.0 \mathrm{mU} / \mathrm{l}$ are appropriate, but TSH $<0.1$ is also acceptable if the patient is doing well clinically and serum $\mathrm{FT}_{4}$ is in the appropriate range.

\section{What are the indications and timing of subtotal thyroidectomy in the management of pregnancy and hyperthyroidism?}

Surgery in pregnancy carries more risks than medical therapy and is complicated by hyperthyroidism. It is associated with an increased risk of spontaneous abortion or premature delivery (45). Thyroidectomy in maternal hyperthyroidism is rarely indicated, and subtotal thyroidectomy is indicated in patients with

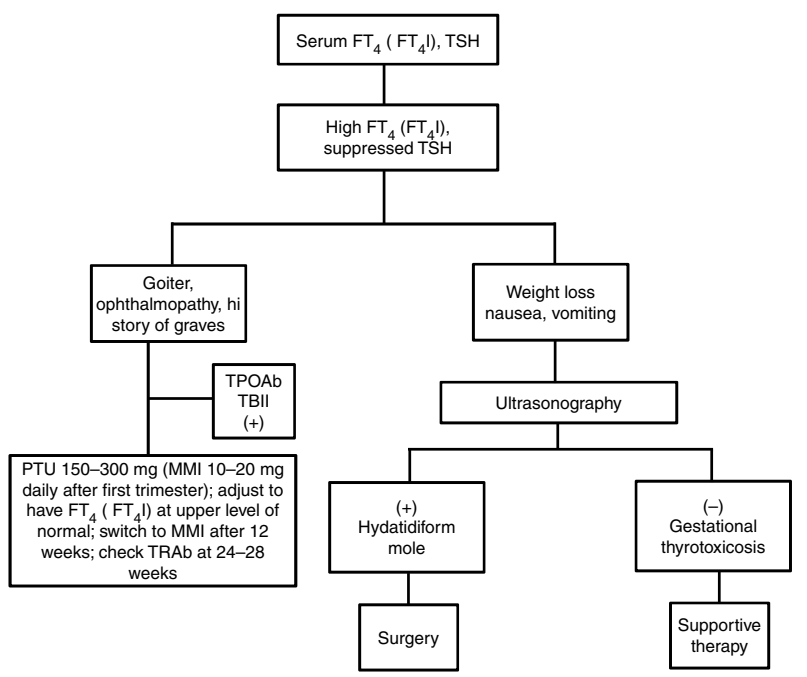

Figure 2 Algorithm for the systematic approach to hyperthyroidism suspected during pregnancy. major or severe adverse reactions to antithyroid drugs, and if hyperthyroidism is uncontrolled because of lack of compliance, high doses of antithyroid drugs are required to control the disease (46) and large goiter that may require high doses of antithyroid drugs (ATD). The optimal timing for surgery is in the second trimester when organogenesis is complete, the uterus is relatively resistant to stimulating events, and the rate of spontaneous miscarriage is reduced.

\section{Should ATD therapy be discontinued in euthyroid pregnant women during the third trimester of gestation?}

Some clinicians recommend discontinuation of antithyroid medications in the third trimester in $20-30 \%$ of pregnant women who have been euthyroid for several weeks on small doses and have low TRAb titers. A study has shown more recurrence of hyperthyroidism in the post partum period in those who had stopped antithyroid therapy compared with those who had continued such treatment throughout pregnancy and post partum (47).

\section{What is the appropriate management of neonatal thyrotoxicosis?}

Neonatal hyperthyroidism is due to transplacental transfer of maternal TRAb and occurs in $5 \%$ of neonates of mothers with Graves' disease (13). $\mathrm{FT}_{4}$ and TSH should be measured in the cord blood of any infant delivered by women with a history of Graves' disease. If the woman was treated with ATD upto the end of pregnancy, clinical manifestations of neonatal hyperthyroidism may be only seen for the first time a few days after delivery, because the fetus was protected by the ATD received from the mother during the final weeks of gestation. Antithyroid treatment and propranolol should be initiated. Either MMI $0.5-1 \mathrm{mg} / \mathrm{kg}$ or PTU $5-10 \mathrm{mg} / \mathrm{kg}$ daily should be given to neonates with hyperthyroidism. Propranolol $2 \mathrm{mg} / \mathrm{kg}$ daily is helpful to slow down pulse rate and reduce hyperactivity in ill neonates. Lugol solution or potassium iodide and glucocorticoids may also be given in more severe cases (48).

\section{What is the best management of hyperthy- roidism in the post partum period?}

Cases of thyrotoxicosis due to Graves' disease occur more frequently during the post partum period than at other times in women of childbearing age (49). In the months following delivery, exacerbation of immune reactivity occurs between 3 and 12 months post partum (50). Therefore, autoimmune thyroid disorders may begin to recur or exacerbate during this crucial period. Graves' disease and post partum thyroiditis are two major causes of thyrotoxicosis in the first year after delivery. 
Thyrotoxicosis caused by post partum thyroiditis usually does not require treatment; therefore, it is of utmost importance to differentiate between Graves' disease and post partum thyroiditis, TSH receptor antibodies being positive in the former and negative in the latter (51). If a woman is not breastfeeding, radioiodine uptake may show low values in post partum thyroiditis and elevated or normal values in Graves' disease. Antithyroid drugs are the mainstay of treatment for thyrotoxicosis during post partum period (52). Neither PTU nor MMI causes any alterations in thyroid function and physical and mental development of infants breast-fed by lactating thyrotoxic mothers (53-55). Methimazole is the preferred drug, because of a risk of potential hepatotoxicity of PTU in either mother or child (56).

\section{Conclusion}

Management of hyperthyroidism during pregnancy and lactation requires special considerations and should be carefully implemented to prevent any adverse effects on the mother, fetus, or neonate. Taking into account the probability of association of fetal teratogenicity with MMI, PTU is recommended as the drug of choice during the first trimester of pregnancy, but due to its hepatotoxicity, it should be changed to MMI thereafter. Surgery is considered only if large doses of antithyroid drugs could not control hyperthyroidism or serious drug side effects have occurred. Radioiodine therapy is contraindicated during pregnancy and lactation. During the post partum period, all antithyroid drugs could be administered in nursing mothers, but MMI should be the drug of choice because of the risk of potential hepatotoxicity of PTU in either mother or child.

\section{Declaration of interest}

The authors declare that there is no conflict of interest that could be perceived as prejudicing the impartiality of the research reported.

\section{Funding}

This research did not receive any specific grant from any funding agency in the public, commercial, or not-for-profit sector.

\section{References}

1 Mestman JH. Hyperthyroidism in pregnancy. Clinical Obstetrics and Gynecology 199740 45-64. (doi:10.1097/00003081-199703 000-00007)

2 Burrow GN. Thyroid function and hyperfunction during gestation. Endocrine Reviews 199314 194-202. (doi:10.1210/edrv-14-2194)

3 Davis LE, Lucas MJ, Hankins GD, Roark ML \& Cunningham FG. Thyrotoxicosis complicating pregnancy. International Journal of Gynecology and Obstetrics 198930 83. (doi:10.1016/0020-7292 (89)90248-8)
4 Lazarus JH. Thyroid disorders associated with pregnancy. Treatments in Endocrinology 20054 31-41. (doi:10.2165/00024 677-200504010-00004)

5 Hershman JM. Human chorionic gonadotropin and the thyroid: hyperemesis gravidarum and trophoblastic tumors. Thyroid 1999 9 653-657. (doi:10.1089/thy.1999.9.653)

6 Tan JY, Loh KC, Yeo GS \& Chee YC. Transient hyperthyroidism of hyperemesis gravidarum. British Journal of Obstetrics and Gynaecology 2002109 683-688. (doi:10.1111/j.1471-0528. 2002.01223.x)

7 Mestman JH. Hyperthyroidism in pregnancy. Best Practice and Research. Clinical Endocrinology and Metabolism 200418 267-288. (doi:10.1016/j.beem.2004.03.005)

8 Phoojaroenchanachai M, Sriussadaporn S, Peerapatdit T, Vannasaeng S, Nitiyanant W, Boonnamsiri V \& Vichayanrat A. Effect of maternal hyperthyroidism during late pregnancy on the risk of neonatal low birth weight. Clinical Endocrinology $2001 \mathbf{5 4}$ 365-370. (doi:10.1046/j.1365-2265.2001.01224.x)

9 Papendieck P, Chiesa A, Prieto L \& Gruñeiro-Papendieck L. Thyroid disorders of neonates born to mothers with Graves' disease. Journal of Pediatric Endocrinology and Metabolism 2009 22 547-553. (doi:10.1515/JPEM.2009.22.6.547)

10 Stagnaro-Green A. Maternal thyroid disease and preterm delivery. Journal of Clinical Endocrinology and Metabolism 200994 21-25. (doi:10.1210/jc.2008-1288)

11 Glinoer D. The regulation of thyroid function in pregnancy: pathways of endocrine adaptation from physiology to pathology. Endocrine Reviews 199718 404-433. (doi:10.1210/er.18.3.404)

12 ACOG Practice Bulletin. Clinical management guidelines for obstetrician-gynecologists. Number 37, August 2002. (Replaces Practice Bulletin Number 32, November 2001).

13 Laurberg P, Nygaard B, Glinoer D, Grussendorf M \& Orgiazzi J. Guidelines for TSH-receptor antibody measurements in pregnancy: results of an evidence-based symposium organized by the European Thyroid Association. European Journal of Endocrinology 1998139 584-586. (doi:10.1530/eje.0.1390584)

14 Weetman AP. Graves' disease. New England Journal of Medicine 2000343 1236-1248. (doi:10.1056/NEJM200010263431707)

15 Ecker JL \& Musci TJ. Thyroid function and disease in pregnancy. Current Problems in Obstetrics, Gynecology and Fertility 200023 109-122.

16 Mitsuda N, Tamaki H, Amino N, Hosono T, Miyai K \& Tanizawa O. Risk factors for developmental disorders in infants born to women with Graves disease. Obstetricia et Gynecologica $199280359-364$.

17 Momotani N, Ito K, Hamada N, Ban Y, Nishikawa Y \& Mimura T. Maternal hyperthyroidism and congenital malformation in the offspring. Clinical Endocrinology 198420 695-700. (doi:10.1111/ j.1365-2265.1984.tb00119.x)

18 Azizi F. The safety and efficacy of antithyroid drugs. Expert Opinion on Drug Safety 20065 107-116. (doi:10.1517/14740338.5.1. 107)

19 Mandel SJ, Brent GA \& Larsen PR. Review of antithyroid drug use during pregnancy and report of a case of aplasia cutis. Thyroid 19941 129-133. (doi:10.1089/thy.1994.4.129)

20 Karras S, Tzotzas T, Kaltsas T \& Krassas GE. Pharmacological treatment of hyperthyroidism during lactation: review of the literature and novel data. Pediatric Endocrinology Reviews: PER $2010825-33$.

21 Redmond GP. Propranolol and fetal growth retardation. Seminars in Perinatology 19826 142-147.

22 Krassas GE, Poppe K \& Glinoer D. Thyroid function and human reproductive health. Endocrine Reviews 201031 702-755. (doi:10.1210/er.2009-0041)

23 Mortimer RH, Cannell GR, Addison RS, Johnson LP, Roberts MS \& Bernus I. Methimazole and propylthiouracil equally cross the perfused human term placental lobule. Journal of Clinical Endocrinology and Metabolism 199782 3099-3102. (doi:10. $1210 /$ jc.82.9.3099)

24 Gardner DF, Cruikshank DP, Hays PM \& Cooper DS. Pharmacology of propylthiouracil (PTU) in pregnant hyperthyroid women: 
correlation of maternal PTU concentrations with cord serum thyroid function tests. Journal of Clinical Endocrinology and Metabolism 198662 217-220. (doi:10.1210/jcem-62-1-217)

25 Momotani N, Noh JY, Ishikawa N \& Ito K. Effects of propylthiouracil and methimazole on fetal thyroid status in mothers with Graves' hyperthyroidism. Journal of Clinical Endocrinology and Metabolism 199782 3633-3636. (doi:10.1210/jc.82.11.3633)

26 Aloush V, Litinsky I, Caspi D \& Elkayam O. Propylthiouracilinduced autoimmune syndromes: two distinct clinical presentations with different course and management. Seminars in Arthritis and Rheumatism 200636 4-9. (doi:10.1016/j.semarthrit.2006. 03.003)

27 Noh JY, Yasuda S, Sato S, Matsumoto M, Kunii Y, Noguchi Y, Mukasa K, Ito K, Ito K, Sugiyama O, Kobayashi H, Nihojima S, Okazaki M \& Yokoyama S. Clinical characteristics of myeloperoxidase antineutrophil cytoplasmic antibody-associated vasculitis caused by antithyroid drugs. Journal of Clinical Endocrinology and Metabolism 200994 2806-2811. (doi:10.1210/jc.2008-2700)

28 Liaw YF, Huang MJ, Fan KD, Li KL, Wu SS \& Chen TJ. Hepatic injury during propylthiouracil therapy in patients with hyperthyroidism. A cohort study. Annals of Internal Medicine 1993118 424-428.

29 Rivkees SA \& Mattison DR. Propylthiouracil (PTU) hepatoxicity in children and recommendations for discontinuation of use. International Journal of Pediatric Endocrinology 20092009132041. (doi:10.1155/2009/132041)

30 Russo MW, Galanko JA, Shrestha R, Fried MW \& Watkins P. Liver transplantation for acute liver failure from drug induced liver injury in the United States. Liver Transplantation 200410 1018-1023. (doi:10.1002/lt.20204)

31 Cooper DS \& Rivkees SA. Putting propylthiouracil in perspective. Journal of Clinical Endocrinology and Metabolism $2009 \mathbf{9 4}$ 1881-1882. (doi:10.1210/jc.2009-0850)

32 Morris CV, Goldstein RM, Cofer JB, Solomon H \& Klintmalm GB. An unusual presentation of fulminant hepatic failure secondary to propylthiouracil therapy. Clinical Transplants, 1989311.

33 Parker WA. Propylthiouracil-induced hepatotoxicity. Clinical Pharmacology 19821 1471-1474.

34 Martínez-Frías ML, Cereijo A, Rodríguez-Pinilla E \& Urioste M. Methimazole in animal feed and congenital aplasia cutis. Lancet 1992339 742-743. (doi:10.1016/0140-6736(92)90640-0)

35 Nachum Z, Rakover Y, Weiner E \& Shalv E. Graves' disease in pregnancy: prospective evaluation of a selective invasive treatment protocol. American Journal of Obstetrics and Gynecology 2003 189 159-165. (doi:10.1067/mob.2003.321)

36 Van Dijke CP, Heydendael RJ \& De Kleine MJ. Methimazole, carbimazole, and congenital skin defects. Annals of Internal Medicine 1987106 60-61.

37 Clementi M, Di Gianantonio E, Pelo E, Mammi I, Basile RT \& Tenconi R. Methimazole embryopathy: delineation of the phenotype. American Journal of Medical Genetics 199983 43-46. (doi:10. 1002/(SICI)1096-8628(19990305)83:1 < 43::AID-AJMG8 > 3.0. $\mathrm{CO} ; 2-\mathrm{C})$

38 Di Gianantonio E, Schaefer C, Mastroiacovo PP, Cournot MP, Benedicenti F, Reuvers M, Occupati B, Robert E, Bellemin B, Addis A, Arnon J \& Clementi M. Adverse effects of prenatal methimazole exposure. Teratology 200164 262-266. (doi:10. 1002/tera.1072)

39 Clementi M, Di Gianantonio E, Cassina M, Leoncini E, Botto LD, Mastroiacovo P \& SAFE-Med Study Group. Treatment of hyperthyroidism in pregnancy and birth defects. Journal of Clinical Endocrinology and Metabolism 201095 E337-E341. (doi:10. 1210/jc.2010-0652)

40 Barbero P, Valdez R, Rodrguez H, Tiscornia C, Mansilla E, Allons A, Coll S \& Liascovich R. Choanal atresia associated with maternal hyperthyroidism treated with methimazole: a case-control study. American Journal of Medical Genetics 2008 146A 2390-2395. (doi:10.1002/ajmg.a.32497)
41 Momotani N, Noh J, Oyanagi H, Ishikawa N \& Ito K. Antithyroid drug therapy for Graves' disease during pregnancy. Optimal regimen for fetal thyroid status. New England Journal of Medicine 1986315 24-28. (doi:10.1056/NEJM198607033150104)

42 Cheron RG, Kaplan MM, Larsen PR, Selenkow HA \& Crigler JF Jr. Neonatal thyroid function after propylthiouracil therapy for maternal Graves' disease. New England Journal of Medicine 1981 304 525-528. (doi:10.1056/NEJM198102263040907)

43 Eisenstein Z, Weiss M, Katz Y \& Bank H. Intellectual capacity of subjects exposed to methimazole or propylthiouracil in utero. European Journal of Pediatrics 1992151 558-559. (doi:10.1007/ BF01957719)

44 Azizi F, Khamseh ME, Bahreynian M \& Hedayati M. Thyroid function and intellectual development of children of mothers taking methimazole during pregnancy. Journal of Endocrinological Investigation 200225 586-589.

45 Roti E, Minelli R, Gardini E \& Braverman LE. Controversies in the treatment of thyrotoxicosis. Advances in Endocrinology and Metabolism 19945429.

46 Brodsky JB, Cohen EN, Brown BW, Wu ML \& Whitcher C. Surgery during pregnancy and fetal outcome. American Journal of Obstetrics and Gynecology 1980138 1165-1167.

47 Nakagawa Y, Mori K, Hoshikawa S, Yamamoto M, Ito S \& Yoshida K. Postpartum recurrence of Graves' hyperthyroidism can be prevented by the continuation of antithyroid drugs during pregnancy. Clinical Endocrinology 200257 467-471. (doi:10. $1046 / j .1365-2265.2002 .01615 . x)$

48 Radetti G, Zavallone A, Gentili L, Beck-Peccoz P \& Bona G. Fetal and neonatal thyroid disorders. Minerva Pediatrica 200254 383-400.

49 Azizi F. Effect of methimazole treatment of maternal thyrotoxicosis on thyroid function in breast-feeding infants. Journal of Pediatrics 1996128 855-857. (doi:10.1016/S0022-3476(96)70342-6)

50 Amino N, Tanizawa O, Mori H, Iwatani Y, Yamada T, Kurachi K, Kumahara Y \& Miyai K. Aggravation of thyrotoxicosis in early pregnancy and after delivery in Graves' disease. Journal of Clinical Endocrinology and Metabolism 198255 108-112. (doi:10.1210/ jcem-55-1-108)

51 Pearce EN, Farwell AP \& Braverman LE. Thyroiditis. New England Journal of Medicine $2003 \mathbf{3 4 8}$ 2646-2655. (doi:10.1056/ NEJMra021194)

52 Azizi F. Treatment of post-partum thyrotoxicosis. Journal of Endocrinological Investigation 200629 244-247.

53 Azizi F, Khoshniat M, Bahrainian M \& Hedayati M. Thyroid function and intellectual development of infants nursed by mothers taking methimazole. Journal of Clinical Endocrinology and Metabolism 200085 3233-3238. (doi:10.1210/jc.85.9.3233)

54 Azizi F, Bahrainian M, Khamseh M \& Khoshniat M. Intellectual development and thyroid function in children who were breast-fed by thyrotoxic mothers taking methimazole. Journal of Pediatric Endocrinology and Metabolism 200316 1239-1243.

55 Azizi F. Thyroid function in breast-fed infants is not affected by methimazole-induced maternal hypothyroidism: results of a retrospective study. Journal of Endocrinological Investigation 2003 26 301-304.

56 Bahn RS, Burch HS, Cooper DS, Garber JR, Greenlee CM, Klein IL, Laurberg P, McDougall IR, Rivkees SA, Ross D, Sosa JA \& Stan MN. The role of propylthiouracil in the management of Graves' disease in adults: report of a meeting jointly sponsored by the American Thyroid Association and the Food and Drug Administration. Thyroid 200919 673-674. (doi:10.1089/thy.2009.0169)

Received 25 January 2011

Accepted 9 March 2011 